\title{
Synthesis of 3-Tetrazolylmethyl-4H-Chromen-4-Ones Via Ugi-Azide Reaction Catalyzed by ZnO Nanoparticles ${ }^{\dagger}$
}

\author{
Manuel A. Rentería-Gómez and Rocío Gámez-Montaño * \\ Departamento de Química, Universidad de Guanajuato, Noria Alta S/N, Col. Noria Alta, \\ Guanajuato 36050, Mexico; vmxrntclonealex@gmail.com \\ * Correspondence: rociogm@ugto.mx; Tel.: +52-473-73-20006 (ext. 8191) \\ + Presented at the 23rd International Electronic Conference on Synthetic Organic Chemistry, \\ 15 November 2019-15 December 2019; Available online: https://ecsoc-23.sciforum.net/.
}

Published: 14 November 2019

\begin{abstract}
A serie of novel 3-tetrazolylmethyl-4H-chromen-4-ones were synthesized via Ugi-azide reaction under mild ultrasound-assisted conditions (room temperature, $\mathrm{EtOH}, 10 \% \mathrm{~mol} \mathrm{ZnO}$ nanoparticles). The products containing two privileged heterocyclic frameworks: 1,5- disubstituted$1 H$-tetrazole and 3-substituted-4H-chromen chromen-4-ones, which are present in a variety of bioactive compounds and commercial drugs
\end{abstract}

Keywords: Ugi-azide; chromone; ultrasound-assisted; ZnO nanoparticles

\section{Introduction}

Chromones are benzoannelated $\gamma$-pyrone (4H-chromen-4-one, 4H-1-benzopyran-4-one) heterocycle present in numerous natural and synthetic products is recognized as a privileged structure and a useful template for the design of novel compounds with potential pharmacological interest. This scaffold showing interesting biological activities such as anti-inflammatory, anticancer, anti-oxidant, and anti-microbial activities (Figure 1) [1-3].
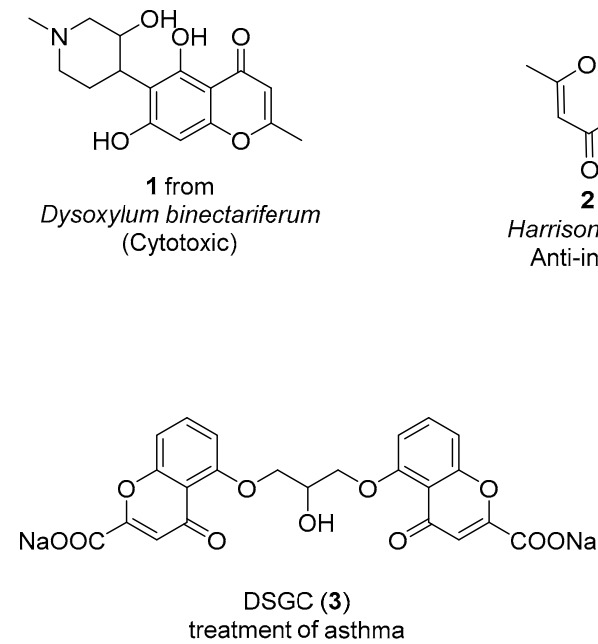

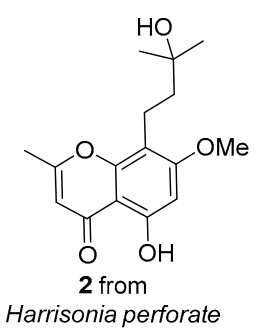

Anti-inflamatory

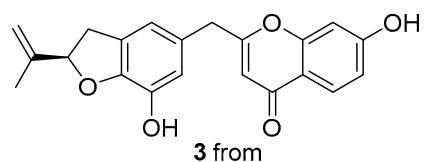

Crotalaria albida Antidyslipidemic
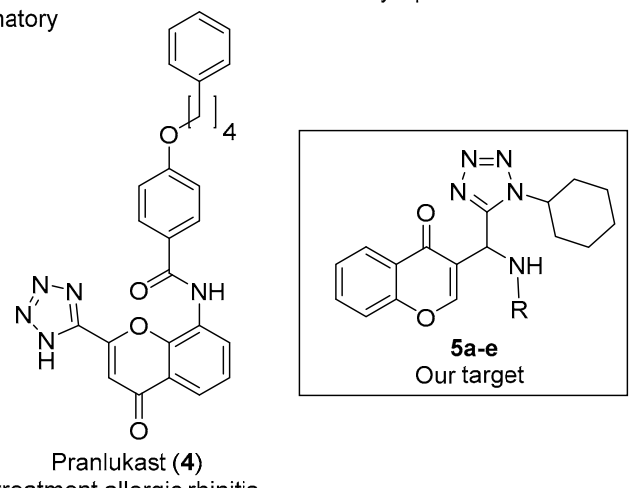

treatment allergic rhinitis

Figure 1. Some natural and synthetic chromones. 
Additionally, 1,5-disubstituted-tetrazoles (1,5-DS-T) are bioisosteres of the cis-amide bond in peptides due to their similar physochemical properties in living systems. mimicking their bioactive conformations, for this reason are a privileged class of heterocycles of high interest in medicinal chemistry [4].

In this context, the interest of synthetized of bis-heterocycles containing the 1,5-DS-T moiety lies in their pharmacophoric features and in the ability to improve pharmacokinetic and pharmacodynamic properties like the increase of metabolic resistance and decrease of toxicity [5].

The main rutes for the synthesis of 1,5-DS-T are the [2+3] intermolecular cycloaddition and the Ugi-azide (UA) reaction in which the carboxylic acid is replaced by hydrazoic acid in the classical Ugi reaction [6].

The isocyanide-based multicomponent reaction (IMCR) is a powerful tool that plays a central role in the synthesis of heterocycles. In this context, the UA reaction coupling with suitably postcondensation processes have been used to access tetrazoles containing heterocyclic scaffolds allows increase structural complexity [4].

In recent years ultrasound irradiation (USI) has gained more attention in modern synthetic chemistry. The reactions by USI can be accelerates the rate of reaction and reduce reaction times at frecuently took place at ambient temperature in mild conditions. The use of USI in isocyanide-based multicomponent reaction (IMCR) is little reported [7-14].

In this work, we describe the first ultrasound assisted synthesis of 3-tetrazolylmethyl-4Hchromen-4-ones via the UA reaction under mild conditions. The change of indium trichloride as catalyst by $\mathrm{ZnO}$ nanoparticles is an useful alternative with low cost to carry out the reaction under mild conditions (Scheme 1) [15,16].

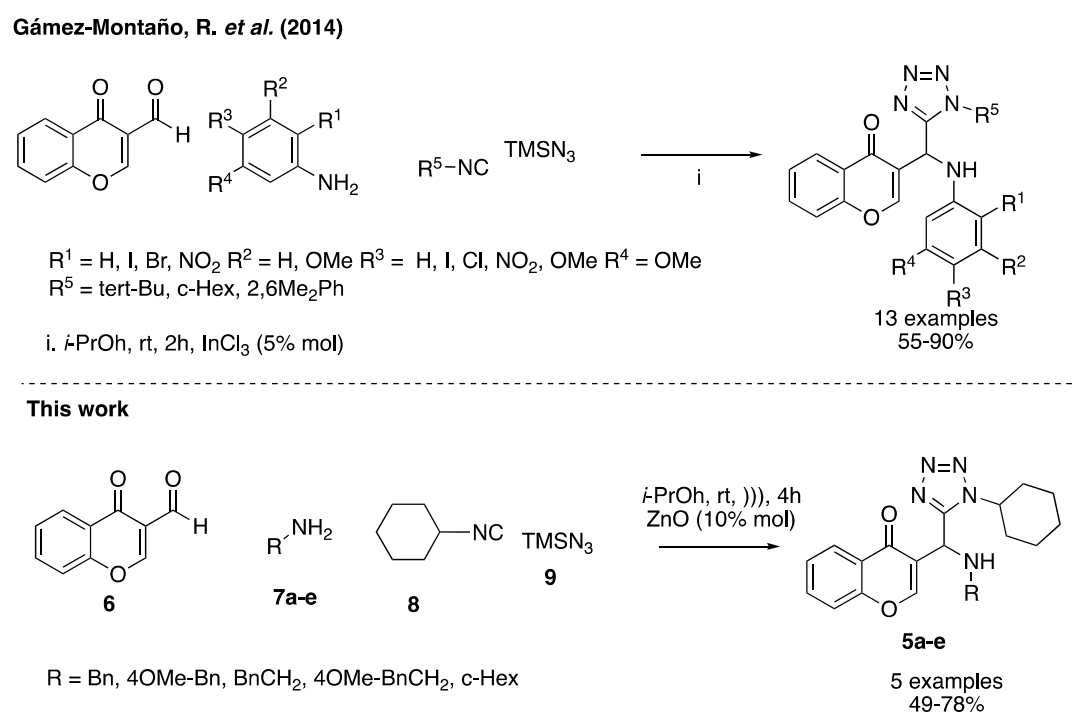

Scheme 1. Previous report and this work.

\section{Results and Discussion}

In order to develop conditions for the UA reaction, we started the synthesis of 3tetrazolylmethyl-4H-chromen-4-ones analogue $5 \mathbf{a}$ by reacting 3 -formylchromone, benzylamine, cyclohexyl isocyanide (8) and azidotrimethylsilane (9). Initially we performed the UA under classical conditions in $\mathrm{MeOH}$ at room temperature, the 5a product was generated in 37\% after $12 \mathrm{~h}$ (Entry 1, Table 1). When the reaction was performed in ethanol as solvent the product $\mathbf{5 a}$ was generated in $33 \%$ after $12 \mathrm{~h}$ (Entry 2). Changing the solvent to isopropanol better results were obtained, $5 \mathrm{a}$ was obtained in $46 \%$. The use of USI (Entries 4-5) resulted in better yields of $45 \%$ and $78 \%$, after 1 and $4 \mathrm{~h}$, respectively. Performing the reaction at $60{ }^{\circ} \mathrm{C}$ the yield was of $76 \%$, the reaction proceeds well at room temperature (Entry 6). The use of $\mathrm{NH}_{4} \mathrm{Cl}$ and $\mathrm{TsOH}$ as catalysts resulted in lower yields of $39 \%$ and $49 \%$, respectively (Entries 8-9, Table 1). 
Table 1. Reaction optimizing conditions 5 a.

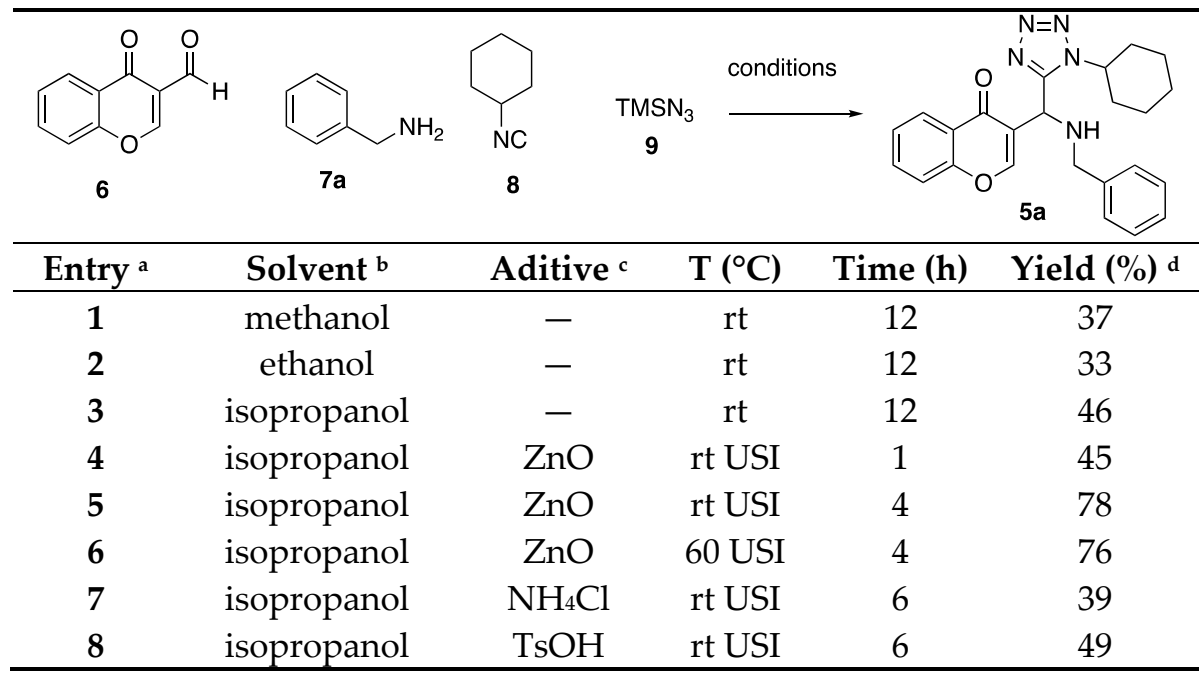

a Reactions performed with 1.0 equiv. 3-formylchromone, 1.0 equiv. of benzylamine , 1.0 equiv. of cyclohexyl isocyanide (8) and 1.0 equiv. of azidotrimethylsilane $(9){ }^{\mathrm{b}}[0.5 \mathrm{M}]^{\mathrm{c}} 10 \% \mathrm{~mol}$. ${ }^{\mathrm{d}}$ Isolated yield. r.t. = room temperature.

Using our optimized conditions, we synthesized the series of 3-tetrazolylmethyl-4H-chromen-4ones (5a-e) shown in Scheme 2. The versatility of the developed methodology was examined using different amines as benzyl, 4-methoxybenzyl, phenethyl, 4-methoxyphenethyl and cyclohexyl (8a-f). The respective products $\mathbf{5 a - f}$ were obtained in moderate to good yields (40-79\%).

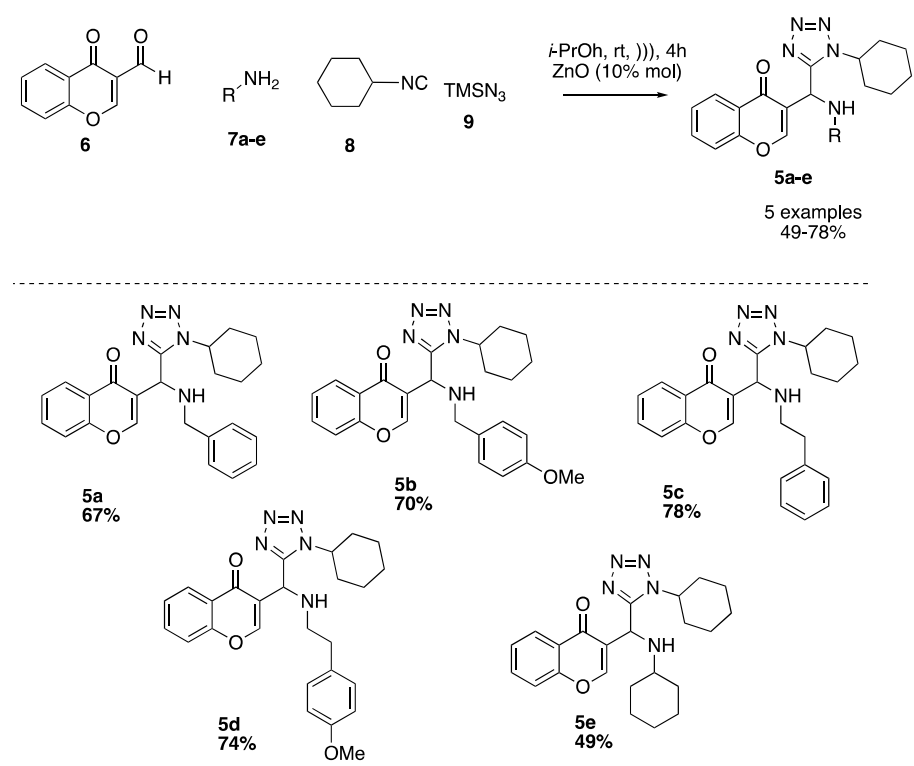

Scheme 2. Sustrate scope.

A plausible reaction mechanism is proposed in the Scheme 3 and two suggested pathways for the activation of the imine are presented. 


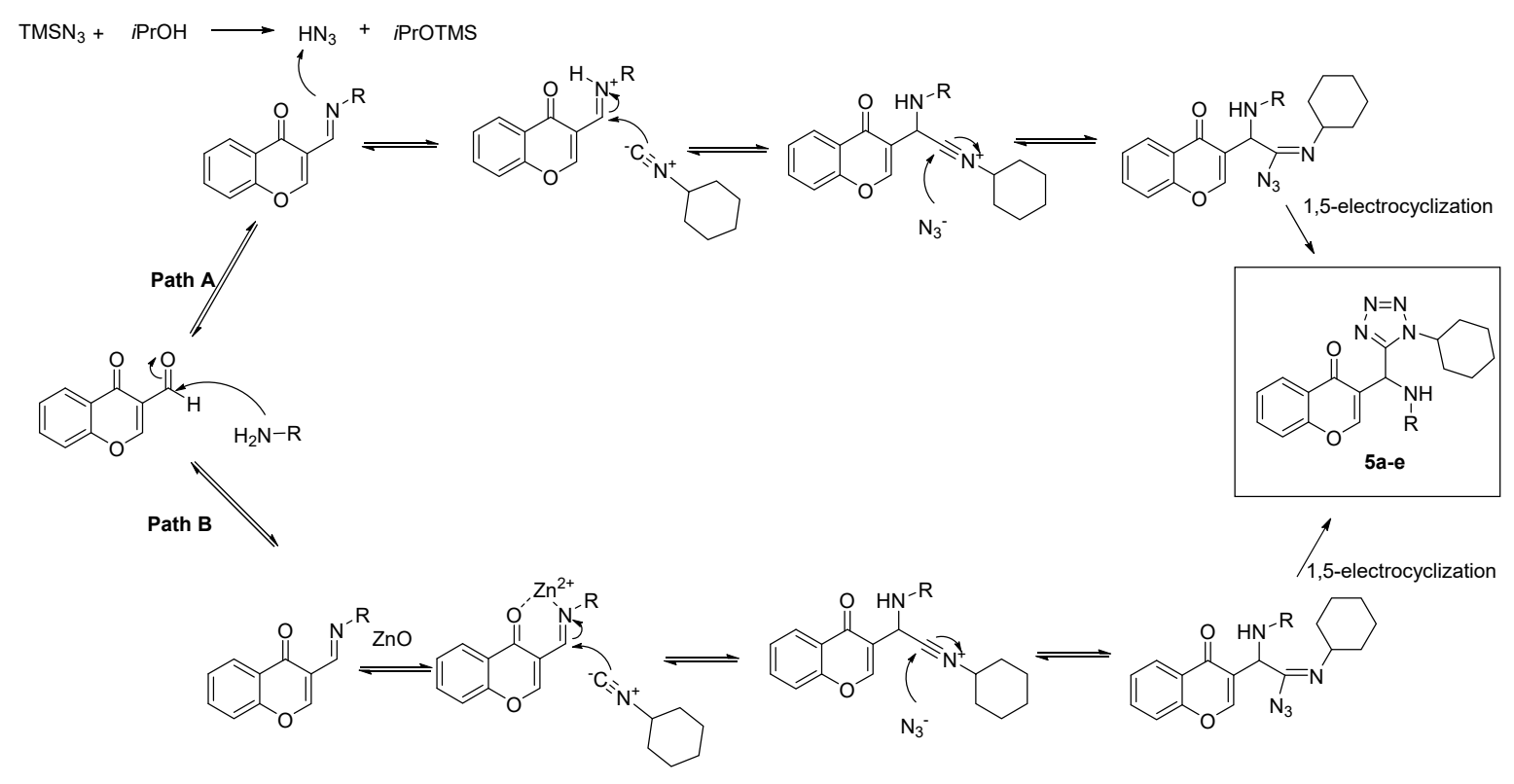

Scheme 3. Plausible Ugi-azide mechanims 5a.

\section{Experimental Section}

\subsection{General Information, Instrumentation, and Chemicals}

${ }^{1} \mathrm{H}$ and ${ }^{13} \mathrm{C}$ NMR spectra were acquired on Bruker Avance III spectrometers (500 or $400 \mathrm{MHz}$ ). The solvent used was deuterated chloroform $\left(\mathrm{CDCl}_{3}\right)$. Chemical shifts are reported in parts per million ( $\delta / \mathrm{ppm})$. The internal reference for ${ }^{1} \mathrm{H}$ NMR spectra is trimethylsilane at $0.0 \mathrm{ppm}$. The internal reference for ${ }^{13} \mathrm{C}$ NMR spectra is $\mathrm{CDCl}_{3}$ at $77.0 \mathrm{ppm}$. Coupling constants are reported in Hertz $(\mathrm{J} / \mathrm{Hz})$. Multiplicities of the signals are reported using the standard abbreviations: singlet (s), doublet (d), triplet (t), quartet (q), and multiplet (m). NMR spectra were analyzed using the MestreNova software version 10.0.1-14719. IR spectra were acquired on a Perkin Elmer 100 spectrometer using an Attenuated Total Reflectance (ATR) method with neat compounds. The absorbance peaks are reported in reciprocal centimeters $\left(v_{\max } / \mathrm{cm}^{-1}\right)$. Reaction progress was monitored by Thin-Layer Chromatography (TLC) on precoated silica-gel $60 \mathrm{~F}_{254}$ plates and the spots were visualized under UV light at 254 or $365 \mathrm{~nm}$. Mixtures of hexane with ethyl acetate (EtOAc) were used to run TLC and for measuring retention factors $\left(R_{f}\right)$. Flash column chromatography was performed using silica gel (230-400 mesh) and mixtures of hexane with EtOAc in different proportions $(v / v)$ as the mobile phase. All reagents were purchased from Sigma-Aldrich and were used without further purification. Chemical names and drawings were obtained using the ChemBioDraw Ultra 13.0.2.3020 software package. The purity for all the synthesized products (up to 99\%) was assessed by NMR.

\subsection{Synthesis and Characterization of the 3-Tetrazolylmethyl-4H-Chromen-4-Ones 5a-e}

General procedure 1 (GP1): 3-formylchromone (6) (1.0 mmol, 1.0 equiv.) in anhydrous isopropanol with $\mathrm{ZnO}$ nanoparticles $(10 \% \mathrm{~mol})$ at room temperature, corresponding amine (7) (1.0 mmol, 1.0 equiv.), cyclohexyl isocyanides (8) (1.0 mmol, 1.0 equiv.) and azidotrimethylsilane (9) (1.0 mmol, 1.0 equiv.) were sequentially added in a $10 \mathrm{~mL}$ sealed vial. The resulting mixture was sonicated at room temperature $(45 \mathrm{kHz})$ for $4 \mathrm{~h}$. Then, the solvent was removed to dryness and the crude was purified by silica-gel column chromatography to afford the products $\mathbf{5 a}-\mathbf{f}$.

3-((benzylamino)(1-cyclohexyl-1H-tetrazol-5-yl)methyl)-4H-chromen-4-one (5a)

According to GP1, 3-formylchromone (40 mg, $0.230 \mathrm{mmol}), \mathrm{ZnO}(1.87 \mathrm{mg}, 0.22 \mathrm{mmol})$, benzylamine $\left(\begin{array}{lllllll}25.0 & \mu \mathrm{L} & 0.230 & \mathrm{mmol}\end{array}\right)$, azidotrimethylsilane $(29.0 \mu \mathrm{L}, 0.230 \mathrm{mmol})$ and 
cyclohexylisocyanide ( $30.0 \mu \mathrm{L}, 0.230 \mathrm{mmol})$ were combined to afford compound $5 \mathrm{a}(64.0 \mathrm{mg}, 67 \%)$ as yellow solid; $\mathrm{Rf}=0.41$ (hexanes-AcOEt, 7:3 v/v) ${ }^{1} \mathrm{H}-\mathrm{NMR}\left(500 \mathrm{MHz}, \mathrm{CDCl}_{3}\right): \delta 8.30(\mathrm{~s}, 1 \mathrm{H}), 8.19$ $(\mathrm{dd}, \mathrm{J}=8.0,1.4 \mathrm{~Hz}, 1 \mathrm{H}), 7.69(\mathrm{ddd}, \mathrm{J}=8.6,7.1,1.7 \mathrm{~Hz}, 1 \mathrm{H}), 7.48-7.40(\mathrm{~m}, 2 \mathrm{H}), 7.22-7.15(\mathrm{~m}, 2 \mathrm{H})$, 6.83-6.76 (m, $1 \mathrm{H}), 6.73-6.68(\mathrm{~m}, 2 \mathrm{H}), 4.98(\mathrm{~d}, \mathrm{~J}=7.1 \mathrm{~Hz}, 1 \mathrm{H}, \mathrm{NH}), 4.75-4.66(\mathrm{~m}, 1 \mathrm{H}), 2.09-1.88$ (m, $8 \mathrm{H}), 1.51-1.38$ (m, $2 \mathrm{H})$; 13C-NMR (126 MHz, CDCl $): \delta 176.7,156.4,155.3,154.5,138.6,134.1$, 129.0, 128.7, 128.3, 127.6, 127.2, 125.8, 125.5, 118.3, 58.0, 51.3, 46.8, 33.1, 32.8, 25.3, 24.9.

3-((1-cyclohexyl-1H-tetrazol-5-yl)((4-methoxybenzyl)amino)methyl)-4H-chromen-4-one (5b)

According to GP1, 3-formylchromone $(40 \mathrm{mg}, 0.230 \mathrm{mmol}), \mathrm{ZnO}(1.87 \mathrm{mg}, 0.22 \mathrm{mmol})$, 4-methoxybenzylamine $(30.0 \mu \mathrm{L}, 0.230 \mathrm{mmol})$, azidotrimethylsilane $(29.0 \mu \mathrm{L}, 0.230 \mathrm{mmol})$ and cyclohexylisocyanide $(30.0 \mu \mathrm{L}, 0.230 \mathrm{mmol})$ were combined to afford compound $\mathbf{5 b}(72.0 \mathrm{mg}, 70 \%)$ as yellow solid; $\mathrm{Rf}=0.28$ (hexanes-AcOEt, 7:3 v/v) ${ }^{1} \mathrm{H}-\mathrm{NMR}\left(500 \mathrm{MHz}, \mathrm{CDCl}_{3}\right): \delta 8.34(\mathrm{~s}, 1 \mathrm{H}), 8.17$ $(\mathrm{dd}, \mathrm{J}=8.1,1.5 \mathrm{~Hz}, 1 \mathrm{H}), 7.69(\mathrm{ddd}, \mathrm{J}=8.6,7.2,1.7 \mathrm{~Hz}, 1 \mathrm{H}), 7.50(\mathrm{~d}, \mathrm{~J}=8.1 \mathrm{~Hz}, 1 \mathrm{H}), 7,44-7.40(\mathrm{~m}, 1 \mathrm{H})$, $7.06(\mathrm{~d}, \mathrm{~J}=8.6 \mathrm{~Hz}, 2 \mathrm{H}), 6.81(\mathrm{~d}, \mathrm{~J}=8.6 \mathrm{~Hz}, 2 \mathrm{H}), 5.44(\mathrm{~s}, 1 \mathrm{H}), 4.54-4.45(\mathrm{~m}, 1 \mathrm{H}), 3.77(\mathrm{~s}, 3 \mathrm{H}), 2.04-1.80$ (m, $6 \mathrm{H}), 1.39-1.27$ (m, $2 \mathrm{H})$; ${ }^{13} \mathrm{C}-\mathrm{NMR}\left(126 \mathrm{MHz}, \mathrm{CDCl}_{3}\right)$ : $\delta$ 176.5, 158.2, 156.3, 154.5, 140.6, 134.1, 131.2, 125.8, 125.5, 123.6, 121.9, 125.8, 125.5, 118.3, 58.8, 58.1, 49.0, 47.8, 35.4, 32.8, 25.4, 24.9, 24.8.

3-((1-cyclohexyl-1H-tetrazol-5-yl)(phenethylamino)methyl)-4H-chromen-4-one (5c)

According to GP1, 3-formylchromone $(40 \mathrm{mg}, 0.230 \mathrm{mmol}), \mathrm{ZnO}(1.87 \mathrm{mg}, 0.22 \mathrm{mmol})$, phenethylamine $(29.0 \mu \mathrm{L}, 0.230 \mathrm{mmol})$, azidotrimethylsilane $(29.0 \mu \mathrm{L}, 0.230 \mathrm{mmol})$ and cyclohexylisocyanide $(30.0 \mu \mathrm{L}, 0.230 \mathrm{mmol})$ were combined to afford compound $5 \mathbf{c}(77.0 \mathrm{mg}, 78 \%)$ as yellow solid; $\mathrm{Rf}=0.39$ (hexanes-AcOEt, 7:3 v/v) ${ }^{1} \mathrm{H}-\mathrm{NMR}\left(500 \mathrm{MHz}, \mathrm{CDCl}_{3}\right): \delta 8.23(\mathrm{~s}, 1 \mathrm{H}), 8.16$ $(\mathrm{dd}, \mathrm{J}=$ 8.0, $1.5 \mathrm{~Hz}, 1 \mathrm{H}), 7.73-7.63(\mathrm{~m}, 1 \mathrm{H}), 7.47(\mathrm{~d}, \mathrm{~J}=8.1 \mathrm{~Hz}, 1 \mathrm{H}), 7.42-7.39(\mathrm{~m}, 1 \mathrm{H})$ 7.39-7.35 $(\mathrm{m}, 1 \mathrm{H}), 7.30-7.23(\mathrm{~m}, 5 \mathrm{H}), 5.44(\mathrm{~s}, 1 \mathrm{H}), 4.54-4.45(\mathrm{~m}, 1 \mathrm{H}), 3.50-3.40(\mathrm{~m}, 2 \mathrm{H}), 3.88-3.84(\mathrm{~m}, 2 \mathrm{H})$, 2.04-1.80 (m, $6 \mathrm{H}), 1.39-1.27$ (m, $2 \mathrm{H})$; ${ }^{13} \mathrm{C}-\mathrm{NMR}\left(126 \mathrm{MHz}, \mathrm{CDCl}_{3}\right):$ : 176.5, 158.2, 156.3, 154.5, 140.6, 134.1, $131.2,125.8,125.5,123.6,121.9,125.8,125.5,118.3,58.8,58.1,49.0,47.8,36.3,35.4,32.8,25.4,24.9,24.8$.

3-((1-cyclohexyl-1H-tetrazol-5-yl)((4-methoxyphenethyl)amino)methyl)-4H-chromen-4-one (5d)

According to GP1, 3-formylchromone (40 mg, $0.230 \mathrm{mmol}), \mathrm{ZnO}(1.87 \mathrm{mg}, 0.22 \mathrm{mmol})$, 4-methoxyphenethylamine $(33.0 \mu \mathrm{L}, 0.230 \mathrm{mmol})$, azidotrimethylsilane $(29.0 \mu \mathrm{L}, 0.230 \mathrm{mmol})$ and cyclohexylisocyanide $(30.0 \mu \mathrm{L}, 0.230 \mathrm{mmol})$ were combined to afford compound $5 \mathbf{d}(78 \mathrm{mg}, 74 \%)$ as yellow solid; $\mathrm{Rf}=0.31$ (hexanes-AcOEt, 7:3 V/V) ${ }^{1} \mathrm{H}-\mathrm{NMR}\left(500 \mathrm{MHz}, \mathrm{CDCl}_{3}\right): \delta 8.23(\mathrm{~s}, 1 \mathrm{H}), 8.16$ $(\mathrm{dd}, \mathrm{J}=8.0,1.5 \mathrm{~Hz}, 1 \mathrm{H}), 7.73-7.63(\mathrm{~m}, 1 \mathrm{H}), 7.47(\mathrm{~d}, \mathrm{~J}=8.1 \mathrm{~Hz}, 1 \mathrm{H}), 7.42-7.39(\mathrm{~m}, 1 \mathrm{H})$ 7.39-7.35 (m, $1 \mathrm{H}), 7.30-7.23(\mathrm{~m}, 5 \mathrm{H}), 5.44(\mathrm{~s}, 1 \mathrm{H}), 4.54-4.45(\mathrm{~m}, 1 \mathrm{H}), 3.50-3.40(\mathrm{~m}, 2 \mathrm{H}), 3.88-3.84(\mathrm{~m}, 2 \mathrm{H})$,), 3.79 (s, 3 H), 2.04-1.80 (m, 6 H), 1.39-1.27 (m, 2 H); ${ }^{13} \mathrm{C}-\mathrm{NMR}\left(126 \mathrm{MHz}, \mathrm{CDCl}_{3}\right): \delta$ 176.5, 158.2, 156.3, 154.5, 140.6, 134.1, 131.2, 125.8, 125.5, 123.6, 121.9, 125.8, 125.5, 118.3, 58.8, 58.1, 49.0, 47.8, 43.9, 36.3, $35.4,32.8,25.4,24.9,24.8$.

3-((1-cyclohexyl-1H-tetrazol-5-yl)(cyclohexylamino)methyl)-4H-chromen-4-one (5e)

According to GP1, 3-formylchromone $(40 \mathrm{mg}, 0.230 \mathrm{mmol}), \mathrm{ZnO}(1.87 \mathrm{mg}, 0.22 \mathrm{mmol})$, cyclohexylamine $(26.0 \mu \mathrm{L}, 0.230 \mathrm{mmol})$, azidotrimethylsilane $(29.0 \mu \mathrm{L}, 0.230 \mathrm{mmol})$ and cyclohexylisocyanide $(30.0 \mu \mathrm{L}, 0.230 \mathrm{mmol})$ were combined to afford compound $\mathbf{5 e}(46 \mathrm{mg}, 49 \%)$ as yellow solid; $\mathrm{Rf}=0.48$ (hexanes-AcOEt, 8:2 v/v) ${ }^{1} \mathrm{H}-\mathrm{NMR}\left(500 \mathrm{MHz}, \mathrm{CDCl}_{3}\right): \delta 8.30(\mathrm{~s}, 1 \mathrm{H}), 8.19$ $(\mathrm{dd}, \mathrm{J}=8.0,1.4 \mathrm{~Hz}, 1 \mathrm{H}), 7.69(\mathrm{ddd}, \mathrm{J}=8.6,7.1,1.7 \mathrm{~Hz}, 1 \mathrm{H}), 6.73-6.68(\mathrm{~m}, 2 \mathrm{H}), 4.79-4.63(\mathrm{~m}, 2 \mathrm{H})$, 2.09-1.38 (m, $20 \mathrm{H}) ;{ }^{13} \mathrm{C}-\mathrm{NMR}\left(126 \mathrm{MHz}, \mathrm{CDCl}_{3}\right): \delta$ 176.7, 156.4, 155.3, 154.5, 138.6, 58.2, 58.0, 51.3, 50.9, $46.8,33.6,33.1,32.3,32.8,25.4,25.3,24.9$.

\section{Conclusions}

In conclusion, we have developed an efficient ultrasound-assisted one-pot Ugi-azide for the synthesis of 3-tetrazolylmethyl-4H-chromen-4-ones, the use of $\mathrm{ZnO}$ is an alternative to $\mathrm{InCl}_{3}$ as catalyst. The main contributions of this work are (a) the design and development of novel synthetic 
strategies based on the Ugi-azide reaction towards complex 1,5-DS-T, and (b) a new I-MCR based methodology to synthesize the finals products under mild conditions.

Author Contributions: All authors contributed equally to this work. All authors have read and agreed to the published version of the manuscript.

Acknowledgments: M.A.R.G. thanks CONACYT for a scholarship (707974/585367). R.G.-M. thanks CONACYT-México (CB-2016-285622) and DAIP Universidad de Guanajuato (CIIC 154/2019) for financial support, the Laboratorio Nacional de Caracterización de Propiedades Fisicoquímícas y Estructura Molecular (CONACYT-México, Project: 123732) for the instrumentation time provided.

Conflicts of Interest: The authors declare no conflict of interest.

\section{References}

1. Nazhand, A.; Durazzo, A.; Lucarini, M.; Romano, R.; Mobilia, M.A.; Izzo, A.A.; Santini, A. Human healthrelated properties of chromones: An overview. Nat. Prod. Res. 2019, 1-16, doi:10.1080/14786419. 2019.1678618.

2. Duan, Y.; Jiang, Y.; Guo, F.; Chen, L.; Xu, L.; Zhang, W.; Liu, B. The antitumor activity of naturally occurring chromones: A review. Fitoterapia 2019, 135, 114-129, doi:10.1016/j.fitote.2019.04.012.

3. Reis, J.; Gaspar, A.; Milhazes, N.; Borges, F. Chromone as a Privileged Scaffold in Drug Discovery: Recent Advances. J. Med. Chem. 2017, 60, 7941-7957, doi:10.1021/acs.jmedchem.6b01720.

4. Herr, R.J. 5-Substituted-1H-tetrazoles as carboxylic acid isosteres: Medicinal chemistry and synthetic methods. Bioorganic Med. Chem. 2002, 10, 3379-3393, doi:10.1016/s0968-0896(02)00239-0.

5. Ostrovskii, V.A.; Trifonov, R.E.; Popova, E.A. Medicinal chemistry of tetrazoles. Russ. Chem. Bull. 2012, 61, 768-780, doi:10.1007/s11172-012-0108-4.

6. Neochoritis, C.G.; Zhao, T.; Dömling, A. Tetrazoles via Multicomponent Reactions. Chem. Rev. 2019, 119, 1970-2042, doi:10.1021/acs.chemrev.8b00564.

7. Kurva, M.; Pharande, S.G.; Quezada-Soto, A.; Gámez-Montaño, R. Ultrasound assisted green synthesis of bound type bis-heterocyclic carbazolyl imidazo[1,2-a]pyridines via Groebke-Blackburn-Bienayme reaction. Tetrahedron Lett. 2018, 59, 1596-1599, doi:10.1016/j.tetlet.2018.03.031.

8. Claudio-Catalán, M.A.; Pharande, S.G.; Quezada-Soto, A.; Kishore, K.G.; Rentería-Gómez, A.; Padilla-Vaca, F.; Gámez-Montaño, R. Solvent- and Catalyst-Free One-Pot Green Bound-Type Fused Bis-Heterocycles Synthesis via Groebke-Blackburn-Bienaymé Reaction/SNAr/Ring-Chain Azido-Tautomerization Strategy. ACS Omega 2018, 3, 5177-5186, doi:10.1021/acsomega.8b00170.

9. Kaveti, B.; Ramírez-López, S.C.; Gámez Montaño, R. Ultrasound-assisted green one-pot synthesis of linked bis-heterocycle peptidomimetics via IMCR/post-transformation/tandem strategy. Tetrahedron Lett. 2018, 59, 4355-4358, doi:10.1016/j.tetlet.2018.10.062.

10. Shaabani, A.; Hooshmand, S.E. Diversity-oriented catalyst-free synthesis of pseudopeptides containing rhodanine scaffolds via a one-pot sequential isocyanide-based six-component reactions in water using ultrasound irradiation. Ultrason. Sonochem. 2018, 40, 84-90, doi:10.1016/j.ultsonch.2017.06.030.

11. Pharande, S.G.; Corrales Escobosa, A.R.; Gámez-Montaño, R. Endogenous water-triggered and ultrasound accelerated synthesis of 1,5-disubstituted tetrazoles via a solvent and catalyst-free Ugi-azide reaction. Green Chem. 2017, 19, 1259-1262, doi:10.1039/c6gc03324e.

12. Chandgude, A.L.; Narducci, D.; Kurpiewska, K.; Kalinowska-Tłuścik, J.; Dömling, A. Diastereoselective one pot five-component reaction toward 4-(tetrazole)-1,3-oxazinanes. RSC Adv. 2017, 7, 49995-49998, doi:10.1039/c7ra07392e.

13. Unnamatla, M.V.B.; Islas-Jácome, A.; Quezada-Soto, A.; Ramírez-López, S.C.; Flores-Álamo, M.; GámezMontaño, R. Multicomponent One-Pot Synthesis of 3-Tetrazolyl and 3-Imidazo[1,2-a]pyridin Tetrazolo[1,5a]quinolines. J. Org. Chem. 2016, 81, 10576-10583, doi:10.1021/acs.joc.6b01576.

14. Chandgude, A.L.; Dömling, A. An efficient Passerini tetrazole reaction (PT-3CR). Green Chem. 2016, 18, 3718-3721, doi:10.1039/c6gc00910g. 
15. Keshipour, S.; Shaabani, A.; Shojaei, S. Nosrati, H.; Ng S.W. A novel one-pot isocyanide-based three-component reaction: synthesis of highly functionalized imidazo-chromen-4-ones. J. Iran Chem. Soc. 2015, 1655-1663, doi:10.1155/2013/125959.

16. Cano, P.A.; Islas-Jácome, A.; González-Marrero, J.; Yépez-Mulia, L.; Calzada, F.; Gámez-Montaño, R. Synthesis of 3-tetrazolylmethyl-4H-chromen-4-ones via Ugi-azide and biological evaluation against Entamoeba histolytica, Giardia lamblia and Trichomona vaginalis. Bioorganic Med. Chem. 2014, 22, 1370-1376, doi: 10.1007/s13738-015-0640-x

(C) 2019 by the authors. Licensee MDPI, Basel, Switzerland. This article is an open access article distributed under the terms and conditions of the Creative Commons Attribution (CC BY) license (http://creativecommons.org/licenses/by/4.0/). 